\title{
Carl Siedschlag
}

\section{Untersuchungen zur Herausbildung herrschafts- und militärpolitischer Anschauungen bei Leibniz ${ }^{1}$ und Saint-Simon ${ }^{2}$}

\begin{abstract}
Sed propria harum disciplinarum (Ethica / Politica) particulatim exequando praestat conjungere Ethicam et politicam et considerare quomodo maxima in hominibus obtineri possit felicitas. (Leibniz)

Le caractère disparaît du monde. Il n'y a plus que des "épateurs «...Louis XIV-Henri 4. - Saint-Louis - Napoléon - Voltaire - La Bruyère - Saint Simon - ont chacun un goût qui reste - une couleur absolue. Mais tous ces petit agités...Pouah. (Céline, Lettres à Albert Paraz)
\end{abstract}

\section{Vorbemerkung}

"In Constantia cives formavimus ad patiendum et parendum, ita hic eos qui imperant ad regendum ", so lässt sich der niederländische Universalgelehrte Justus Lipsius (1547-1606) zu Beginn der 1589 erschienenen Politicorum sive civilis doctrinae livri sex vernehmen. ${ }^{3}$ Es ist die aus dem persönlichen Erleben der konfessionellen Spaltung und der Bürgerkriegsunruhen hervorgegangene Antwort des Leidener Professors, der als Systematiker des Neustoizismus, als Herausgeber und Kommentator der klassischen Autoren (Tacitus-Ausgabe 1574) bereits in hohem Ansehen stand. ${ }^{4}$

»E Philologia Philosophiam feci« - bekennt Lipsius und meint damit eine nunmehr ethisch-moralisch untermauerte philosophia militans, die, eingewoben in ein zum Bestseller aufgerücktes Lehrbuch, zu einem das frühneuzeitliche Politikverständnis prägenden Wegweiser geriet. ${ }^{5}$ Die mit den Konfessionskriegen einhergehende Auflösung der

1 Fas est ab hoste doceri, 1694.

2 Parallèle des Trois Premiers Rois Bourbons, 1746.

3 Der conseil pédagogique steht vor neuen, die »logique du miroir « überschreitenden Herausforderungen: er bekundet sich »dans un rapport de travail cognitif du réel à savoir «. Robert Damien, Bibliothèque et Etat. Naissance d'une raison politique dans la France du XVIIe siècle, PUF, 1995, S. 297.

4 Jacqueline Lagrée, Juste Lipse, La Restauration du Stoicisme. Etude et traductions de divers traités stoiciens. Collection Philologie et Mercure, Paris 1994.

5 Gerhard Oestreich, Antiker Geist und moderner Staat bei Justus Lipsius (1547-1606). Der Neustoizismus als politische Bewegung. Herausgegeben und eingeleitet von Nicolette Mout, Göttingen 1989. Justus Lipsius, Politica. Six books of Politics or political Instruction. Edited with translation and introduction by Jan Waszink, 2004. Zur eingeforderten Praktikabilität bemerkt Françoise Hildesheimer, Richelieu, 2004, S. 360: »La prudence, c'est une vertu active qui s'applique au domaine de l'action ... c'est donc la vertu politique par excellence «. Siehe dazu Robert 
öffentlichen Ordnung setzte $u$. a. für die Neugestaltung des europäischen Heerwesens eine zuerst in den Niederlanden verwirklichte, sich am römischen Begriff der »disciplina militaris« orientierende Reformbewegung in Gang, die in Lipsius ihren maßgeblichen Befürworter fand: »Disciplina repetenda a Romanis, qui felicissime ea usi ${ }^{6}$ Erfährt im 5. und 6. Buch seiner Politik die "prudence militaire " als eine umfassende Wehr- und Kriegslehre seine besondere Aufmerksamkeit, so hält der Leidener Professor eine dieses Anliegen begründende Rechtfertigung für unumgänglich. Die Beschäftigung insbesondere mit den diesen Fragenkreis berührenden antiken Autoren gründet nicht in der auf ruhige Betrachtung abgestellten Erweiterung des Erkenntnishorizontes eines dem Leben abgewandten, sich dem legere, docere, scribere widmenden Vertreters der späthumanistischen Gelehrtenschicht, wie es seine als typisch gewertete Selbstdarstellung suggeriert. ${ }^{7}$ Vielmehr verkörpert auch Lipsius die »energisch aktiv-praktische Haltung der Generation von 1580/90, die den Grundstein legt zur kommenden Staatsphilosophie«. 8 Auf solcherart praktischer Verwertbarkeit bedacht, lässt Lipsius nicht den Verdacht gelten, ein ihm unvertrautes Gelände, den Estat guerrier zu betreten. Er möchte sich verstanden wissen, als ein durch äußerste Gelehrsamkeit Kundiger, ${ }^{9}$ der die Ausformung

Damien, Bibliothèque et Etat, aaO. (FN 1) S. 301, "La révolution copernicienne" du savoir politique: "S'il (le scribe) n'est plus un philosophe exhortant l'idéal ou mythifiant le réel, il doit devenir un savant informateur, un observateur dont les informations divérsifiés permettront de gouverner un réel peu saisissable«. Eine diese Beobachtungen stützende, an seine Söhne gerichtete väterliche Ermahnung in: Mémoires de Benjamin Aubery de Maurier, Ambassadeur Protestant de Louis XIII (1566-1636). Edités et introduits par C. Martin, Genève 2010, S. 374. »Je fuz d'avis qu'ils reprissent les longues latine et grecque ... et avec ces aydes s'adonner incontinent aprez à quelques bonnes sciences actives zur Erlangung einer honorable profession«. Zum »illustre influence du stoicisme chrétien ... avec en particulier « Juste Lipse et Guillaume du Vair«, aaO. S. $235 f$.

6 Justi Lipsi, Politicorum sive civilis doctrinae libri sex qui Principatum maxime spectant, Wien 1752, V, 13, S. 172; Gerhard Oestreich, »Politischer Neustoizismus und Niederländische Bewegung in Europa und besonders in Brandenburg-Preußen « in: Geist und Gestalt des frübmodernen Staates, Berlin 1969, S. 117ff. Ders.: Derrömische Stoizismus und die oranische Heeresreform, aaO. S. 11ff. Winfried Schulze, »Gerhard Oestreichs Begriff der Sozialdisziplinierung in der frühen Neuzeit « in: ZHF für Historische Forschung, 14. Band, Heft 3, Berlin 1987, S. 286ff. Die Modellhaftigkeit des von Lipsius, du Vair, Charon propagierten "parfait soldat stoicien«, »modèle de la discipline d'Etat «, unterstreicht J. Cornette, Le roi de guerre. Essai sur la souveraineté dans la France du Grand Siècle, 1993, S. $50 \mathrm{ff}$.

7 Siehe: G. Oestreich, »Justus Lipsius in sua re« in: Geist und Gestalt des frübmodernen Staats, aaO. (FN4) S. 80ff.

8 Fritz Neubert, »Die Académie du Palais unter Heinreich III. und die Anfänge der neueren psycho-moralischen Literatur in Frankreich« in: Germanisch-romische Monatsschrift, 21 (1933), S. 456.

9 Gegenüber den auf gloire du Prince, puissance de l'Etat gestimmten Auffassungen macht Crucé (Emery de Lacroix) in seiner 1623 erschienenen Schrift Le nouveau cynée Einwände geltend: »Les coutumes tant anciennes que modernes, ont toujours déféré aux soldats le privilège de noblesse et de commandement, en sorte que l'honneur des autres états et perfections se ternit au lustre éclatant de la vertu militaire.«Siehe Etienne Thuau, Raison d'Etat et pensée politique à l'époque de Richelieu, 1966, S. 154f. 
einer neuen, das Aufkommen des frühmodernen Fürstenstaates stützenden, ${ }^{10}$ auf ethischer Grundlage ruhenden und der discipline militaire unterworfenen Wehridee richtungsweisend und nachhaltig beeinflussen wird.

So erteilt der Deutschland-Emissär Marescot im Rahmen eines im Jahre 1625 verfassten Memoriak Militärfragen berührende Ratschläge, die erkennbar von der prudence militaire neustoischer Provenienz inspiriert wurden. Um dem Misserfolg derjenigen vorzubeugen, die nur halbherzig »cimentent et poursuivent leurs entreprises", verdienen folgende Gesichtspunkte Beachtung: »choix et bonté des capitaines et soldats, discipline militaire", Strafen und Belohnungen "comme leur principale base", regelmäßige Entlohnung, Verpflegung und Ausrüstung. ${ }^{11}$ Auch der secrétaire d'état de la guerre, Le Tellier, als ministre lettré lange unerkannt geblieben, in dessen breitgefächerter Bibliothek neben antiken Autoren auch die Werke von Montaigne, Balzac und Lipsius vertreten sind, ${ }^{12}$ gestaltet seine Militärreform nach anerkannten Leitbildern. Als beispielhaft gilt u. a. die bereits von Ludwig XIII. auf den Weg gebrachte alimentation des troupes. Hierbei auftretende Defizite sind der Moral der Truppe abträglich. "L'bomme de guerre n'a ny respect, ny obeissance s'il n'est bien vestu, bien armé, bien chaussé, bien soul et qu'il n'ait encore quelque chose de reservé en sa bourse. " ${ }^{13}$ Dass das wiederbelebte antike Erfahrungswissen rasch und nachhaltig Schule gemacht hat, nötigt André zu dem durch exemplarische Untersuchungen gestützten, aber den geistesgeschichtlichen Ausgangsort noch nicht benennenden Urteil: »Richelieu, Le Tellier et Louvois ont été au même titre, de , grands vivriersı. «14

10 Über den mit Richelieu freundschaftlich verbundenen Philippe de Cospeau heißt es in einem am 14.11.1602 an Scaliger gerichteten Schreiben des conseiller au Grand Conseil J. Gilot: »Il y a ici un Flamand nommé Cospeau, docteur en théologie, escolier de M. Lipsi, qui a grande réputation, homme de bonne façon, grande mémoire et fort versé en saint Augustin. "Siehe: Tallement des Réaux, Historiettes I, S. 526f. und S. 1156 Anm. 3. Eine eventuelle Vermittlerrolle erscheint überprüfenswert.

11 In: Les Papiers de Richelieu. Section Politique Exterieure, Correspondance et Papiers d'Etat. Edités par Adolf Wild, Tome I, 1616-1629, Empire Allemand, Paris 1982, S. 109.

12 Louis André, Michel Le Tellier et Louvois, Genève 1974, S. 624ff. Olivier Chaline, Le règne de Lowis XIV, 2005, S. 209, unterstreicht ein auf eine »discipline plus rigide, assortie d'une formation professionelle plus systématique« abzielendes Reformbegehren von Le Tellier und Louvois.

13 Lipsius, Les Politiques, aaO. (FN8) S. 170. »Le roi, so lässt sich Richelieu vernehmen, sait bien, que je me suis toujours inquiété des retardements des trésoriers et munitionnaires et que j'ai dit publiquement dans ses conseils que ce n'était rien de mettre des armées sur pied si on me donnait ordre de les faire payer à temps et si on ne pourroyait sérieusement aux vivres. «In: Avenel, Lettres, instructions diplomatiques et papiers d'Etat du Cardinal de Richelieu, V, S. 231. Siehe dazu J. Cornette, Le roi de guerre, aaO. (FN 4) S. $189 f$. und S. 403, Anm. 66.

14 André, aaO. (FN9) S. 377. Zu fortbestehenden Defiziten - »French scholars have not yet paid attention to the links between Lipsius and political theory in the age of Richelieu « - siehe G. Oestreich, Neostoicism and the early modern state, Cambridge, 1982, S. 107. Jean Chagniot, Guerre et Société à l'Epoque moderne, 2001, S. 138f. verweist auf die eine »éthique militaire einschließende vraie leçon morale de Juste Lipse «, übermittelt durch De constantia (1583) und Monita et exempla (1605). Seine Politiklehre erfährt keine besondere Berücksichtigung. 


\section{Gottfried Wilhelm Leibniz (1646-1716), Fas est ab hoste doceri (1694)}

Die von Frankreich ausgehende kriegerische Bedrohung veranlasst Leibniz eine von ihm in deutscher und französischer Sprache abgefasste, seine Autorschaft lange verbergende Schrift zu veröffentlichen, die den Titel trägt: Fas est ab hoste doceri. Quelques Edits de la fabrique du Cardinal Richelieu, pour servir d'exemple comment il faut faire des efforts pour résister à l'ennemi dans un pressant besoin. Avec une Préface adressée à la Nation Allemande et convenable au temps. A Amsterdam MDCXCIV $V^{15}$ - ein derzeit gebotenes Unterfangen "puisque l'Allemagne a plus besoin que jamais des exemples et réflexions qu'il [ce petit ouvrage] contient. "16

Der Beginn des pfälzischen Krieges 1688/89 war für Leibniz Anlass die »Teutsche Nation « auf bereits 1636 ergangene französische Verordnungen zur schnellen, nachhaltig zu betreibenden Mobilmachung aufmerksam zu machen, ein Anliegen, das ihm »bereits eingefallen als Frankreich neulich mit uns öhnversehens gebrochen «. ${ }^{17}$

1692, nach Wiederaufnahme der Beschäftigung mit den »Verordnungen « Richelieus gibt der Autor zu bedenken, ob unter Beachtung des Vorbildes dem feindlichen Angriff mit geballter Kraftanstrengung wirkungsvoller zu begegnen ist. Das erübrigt aber nicht die Frage »ob eine kleine dosis eines vomitorii oder opiati ... zum offtern widerhohlet den Nutzen habe, so die volle gebührende dosis auff einmahl geben kann ${ }^{18}$ Aber den Blick auf die »Staats- und Kriegssachen « richtend, ergeht ein kategorisches Urteil: das »einzige Mittel unser Woblfarth sey, alle Kräffte so viel müglich auff einmahl dranstecken $\ll .{ }^{19}$

Bei der Wahl zwischen einem kostspieligen, aber kurzem Krieg und langanhaltender Versklavung hat die "autorité des grands hommes" und ihr beispielgebendes Handeln Entscheidungshilfe zu leisten. Die zur Vorbereitung einer erfolgversprechenden Militäraktion von Richelieu verfassten und mit "promptitude und ponctualité in die Tat umgesetzten Ordonanzen sind "un bon eschantillon d'une résolution prompte et vigoureuse qui pourroit servir en des pareilles rencontres ". ${ }^{20}$ Ihre als hilfreich erachtete Veröffentlichung möge dazu beitragen die in Sorglosigkeit (nonchalance) verfallene deutsche Nation wachzurütteln um sie durch »bonnes exhortations " vor dem drohenden Verlust ihrer Freiheit zu bewahren.

Solche eindringlichen Ermahnungen sind dem Eingeständnis geschuldet »que nous sommes des gens estranges qui ne scavent jamais tenir le juste milieu«.Von Teilerfolgen

15 In: Gottfried Wilhelm Leibniz, Politische Schriften, Akademie Verlag Berlin 2004, Fünfter Band, 1692-1694, S. 543ff.

$16 \mathrm{AaO}$. (FN13) S. 551. Die Anerkennung des politischen, wirtschaftlichen und militärischen Machtstaatsideals Westeuropas durchdringt, so bemerkt Gerhard Oestreich, allmählich die Mehrheit der bedeutenden Territorien in verschiedener Form und unterschiedlicher Stärke. In: Geist und Gestalt des frühmodernen Staates. Zur Heeresverfassung der deutschen Territorien. Berlin, 1969, S. 303.

$17 \mathrm{AaO}$. (FN13) Einleitung XLIII.

18 AaO. (FN13) S. 543.

$19 \mathrm{AaO}$. (FN13) S. 544.

20 AaO. (FN13) S. 553.

ZfP 59. Jg. 4/2012 
geblendet, breitet sich angesichts des überlegenen Gegners eine den âmes élevées fremde, von Feigheit gezeichnete Mutlosigkeit aus, begleitet von einem trügerischen, gleichgültigen Ruhebedürfnis. Ein solches Fehlverhalten wird auch künftig noch zur Unehre gereichen: "L'bistoire dira, c'est nôtre paresse, nostre peu de courage et nôtre bumeur effeminée qui nous a fait perdre la liberté de la patrie, l'honneur de la nation et la dignité de nôtre etat et famille. «21

Hinsichtlich der der vertu militaire beizumessenden Bedeutung wie sie Lipsius am Beginn seiner Militärlehre umschrieben hat, ist eine Übereinstimmung unverkennbar. Die »courage à l'encontre des ennemis einfordernde vertu militaire est plus excellente que toutes les autres. «22 Aber erst ein ihr geltendes striktes Bemühen ermöglicht ihre Nutzbarmachung. "Il est donc necessaire de l'embrasser à bon escient et doit-on efforcer et de tout son esprit, et de tous ses sens pour parvenir à ceste discipline. «23

Es gilt also, so schlussfolgert Leibniz, ein dem Angreifer standhaltendes bzw. überlegenes militärisches Gegengewicht (supériorité) zu schaffen nach Maßgabe einer das Vorhaben flankierenden prudence très grande, damit anknüpfend an die vielbeachtete Militärdoktrin, der Lipsius die Absicht unterlegt hat »à dresser des preceptes de la guerre universellement ${ }^{2} .24$

Der in Frankreich errungene Vorteil über »dienliche Mittel bey seinen Anschlägen « ${ }^{25}$ $z u$ verfügen - über das Land verteilte Festungen und Waffenarsenale, »Vertrauen bey seinen Soldaten, Gehorsam bey seinen Untertanen, baar Geld« - steht in scharfem Gegensatz zu den Nachteilen seines Gegenübers.

Unentschlossenheit und Missbehagen (refroidissement) unter den durch ausbleibende Erfolge entmutigten Verbündeten, eine Vielzahl von Anführern, nicht untereinander abgestimmte Interessen zeitigen abträgliche Folgen, deren Überwindung großer Anstrengungen bedarf, soll sich nicht der Eindruck verfestigen "que nous sommes des corps sans âme" wenn es gilt das Vaterland zu verteidigen. Frankreich kann nicht allein göttlichen Beistand für sich beanspruchen, er wird denjenigen zuteil »qui se servent bien des lumières et des forces qu'il leur a données, il est pour les bonnes trouppes, pour les sages conseils ${ }^{26}$ So ruft Leibniz dazu auf, in Einigkeit und unter Aufbietung aller Kräfte, »avec corps et biens" dem Wohl des Landes dienlich zu sein.

Vom Irrglauben befreit "que toute la sagesse se trouve renfermée dans la France" gilt es unverzüglich der Auswahl der Führungseliten einen hohen Stellenwert einzuräumen und die Umsetzung der "bons conseils" den "personnes excellentes dans la science militaire" anzuvertrauen. Einem laschen Willen zur Selbstbehauptung - "nostre médiocre conduite - erteilt Leibniz eine Absage und ein unüberlegtes, vorschnelles Handeln "avec une impétuosité avengle" - ist fehl am Platze, denn »la guerre est devenue une

21 AaO. (FN13) S. 557.

22 Les Politiques de Juste Lipse, (FN8) V, 2, S. 158.

$23 \mathrm{AaO}$.

24 Lipsius, aaO. (FN8) S. 155.

25 Leibniz, aaO. (FN13) S. 558.

26 Leibniz aaO. (FN13) S. 561. Siehe dazu: Lipsius, Politik, (FN8) S. 169. 
science " ${ }^{27}$ deren Ausformung der niederländische Universalgelehrte Lipsius Politiklehre maßgeblich vorangetrieben hat. Sie erringt eine sich ausweitende Gültigkeit und lässt ihn als frühmodernen Verfechter einer gemeinsamen, sich allseits in Europa durchsetzenden Wehrverfassung erscheinen "car c'est à elle (l'Europe) que nous parlons et escrivons «. ${ }^{28}$

Die von Leibniz ausschnittartig bezeugten, fortbestehenden Auswirkungen der Niederländischen Bewegung dienen mit Blick auf eine von Saint-Simon in zeitlicher Nähe verfasste, »le fond de sa pensée ou de son paysage ${ }^{29}$ freilegende Charakterstudie als Anregung auf einen vergleichbaren Tatbestand zu verweisen - ablesbar etwa an der Wertschätzung, die dem Begriff der »capacité militaire«in seiner spezifischen Ausrichtung zuteil wird. ${ }^{30}$

\section{Saint-Simon (1675-1755), Parallèle des Trois Premiers Rois Bourbons (1746)}

Die Festellung, dass der stoisierende, lipsianisch geprägte ${ }^{31}$ Moralismus der Geschichtsschreibung bis in das Zeitalter der Aufklärung vorherrschend war, ${ }^{32}$ lässt es geraten erscheinen, das im »Parallèle des trois Rois Bourbons « ${ }^{33}$ seines nur unzulänglich beurteilten

27 Leibniz aaO. (FN13) S. 563. Konkrete Angaben zum "prêcheur suprème de l'évangile de la prudence « bei J. H. Ellioth, Richelieu et Olivarez, 1991, S. 35: Oeuvres Complètes de Lipse in seiner Bibliothèque du Palais-Cardinal, ein Exemplar der »Doctrine Civile « in Rueil vorhanden. Zur »capacité de connaître la gouvernabilité«, in wörtlicher Anlehnung an G. Naudé siehe R. Damien, Bibliothèque et Etat aaO. (FN 1) S. 295: »Ceux qui tiennent le gouvernail des Républiques apuyent leur authorité non seulement par l'assistance de leurs gardes et par le secours d'un grand nombre de gens de guerre mais ... en faisant paroistre de la force et de la constance dans les périls; de l'industrie et de l'adresse dans leurs actions et de la promptitude à les exécuter: et enfin en usant de prudence et de bons Conseils lorsqu'il est question de pourvoir aux accidents qui surviennent.

28 Lipsius, Politik, (FN8) S. 179. Die Überwindung der »histoire bataille « durch Veröffentlichung bislang unbekannten Quellenmaterials ermöglicht im Hinblick auf den militärhistorischen Kenntnisstand (1570-1791) »un premier bilan du renouveau". Siehe Thierry Sarmant, Mars archiviste -Departement de la guerre, Dépôt de la guerre, Archive de la guerre, Band 222, 2001, S. 114.

29 Saint-Simon Traités politiques et autres écrits. Edition établie par Yves Coirault, Bibliothèque de la Pléiade, 1996, Introduction, XV. Die folgenden Zitate sind dieser Ausgabe entnommen.

$30 \mathrm{Zu}$ der in Frankreich durch die littérature morale ausgelösten »fureur du portrait « siehe Dirk van der Cruysse, Le Portrait dans les »Mémoires" $d u$ Duc de Saint-Simon, Paris 1971, S. 32.

31 »Chez Juste Lipse, toute l'énergie mise dans la foi romaine, suppose la sinistre vision d'une impuissance de la foi chrétienne, comme privée de rayonnement, tout l'espoir dans les forces humaines est déployé pour surmonter le désespoir, l'angoisse d'un effondrement de la religion et de l'Europe qu'elle soutient«. Siehe: Christian Mouchel, »Juste Lipse: Un philosophe de transition? « in: Juste Lipse (1547-1606) en son temps. Actes du colloque de Strasbourg, 1994, Paris 1996, Préface S. 18.

32 G. Oestreich, »Politischer Neustoizismus und niederländische Bewegung in Europa und besonders in Brandenburg-Preußen» in: Geist und Gestalt des frühmodernen Staates, Berlin 1969, S. 108.

33 Saint-Simon, Traités politiques, aaO. (FN27).

ZfP 59. Jg. 4/2012 
Favoriten Louis XIII »le Juste", "un monarque qui l'avait fasciné «34 auf weltanschauliche, die "prudence militaire" einschließende Leitvorstellungen, »le fond de sa pensée « 35 hin zu befragen, wobei eine zeittypische Orientierungsgrundlage »la permanence d'une idéologie «36 auch für den »Duc-mémorialiste» Verbindlichkeit beanspruchen darf.

Der zunächst im Rahmen der écrits inédits veröffentlichte Parallèle, ${ }^{37}$ epilogartig eingebettet in das sich vor dem Betrachter ausbreitende »immense fresque historique et passéiste « ${ }^{38}$ möchte sich nicht verstanden wissen als eine die Neugier kaum beflügelnde Wiederholung des bereits vielfach Geschilderten. Der 1746 als "oeuvre tardive" (Faugère) erschienene, "la parfaite maturité $d u$ talent - ou du génie saint-simonien " bezeugende »Parallèle « ${ }^{39}$ beabsichtigt vielmehr das sich hinter einem Schleier von Unwissenheit, Hass und Schmeichelei Verbergende, auf "appréciation méritocratique «40 $\mathrm{ru}-$ hende, jeweils Eigentümliche zum Vorschein zu bringen, »le caractère particulier de chacun des trois monarques ${ }^{41}$ Es ist die Missbilligung der Ungerechtigkeit, die Ludwig XIII. widerfährt - »Roi sans charisme entre Henri IV. et Louis XIV. à côté de Richelieu il est presque un oublié de l'bistoire " ${ }^{2}$ - die Saint-Simon veranlasst ihm gegenüber eine Dankesschuld abzutragen. ${ }^{43}$ So erfährt sein entstelltes Bild durch vergleichende Betrachtung - "la gloire de Louis XIII. lui semblait obscurcie et comme étouffée... les mérites $d u$

34 George Poisson, Monsieur de Saint-Simon, 1973, S. 375. Auf diesem Wege erfüllt sich sein Wunsch im Nachhinein - »qui pensait encore à Louis XIII, hormis notre héros«- eine das Ansehen des »roi de son idéal«schmälernde Gerechtigkeitslücke zu schließen, »une juste remise en place des valeurs «, nicht zuletzt im Hinblick auf die vermeintlich dominierende Persönlichkeit des Kardinalpremiers. Für die »méconnaissance des vertus de son héroique père « macht Yves Coirrault, L'optique de Saint-Simon, 1965, S. 347, Anm. 23, die historiographes de Louis XIV mitverantwortlich.

$35 \mathrm{Zu}$ den Ausformungen von vertu und vice siehe van der Cruysse, aaO. (FN 28) S. 265f. und S. $296 f$.

36 Saint-Simon, Traités politiques, aaO. (FN27) Introduction, S. XI. Den aus einer »boulimie de lecture « sich speisenden, die »érudition classique de l'humanisme" einschließenden »sources de la pensée saint-simonienne « hat Hélène Himelfarb eine erhellende Analyse gewidmet: Saint Simon, lecteur d'histoire et de mémoires. In: XVIIe Siècle, Vol. 94/95, Paris 1971, S. 119f. Zum weiträumigen Leseinteresse: »il avait lu énormément et du meilleur « - die erfassten Bibliotheksbestände weisen auf Vertreter der niederländischen Bewegung hin, siehe Edmond Pognon, Saint-Simon ou l'Observateur véridique. Bibliothèque nationale, 1976, S. XI.

37 Écrits inédits de Saint-Simon, publiés sur les manuscrits conservés au dépôt des Affaires étrangères par P. Faugère, Tome premier, Paris 1880, aaO.

38 Emmanuel Le Roy Ladurie, Saint-Simon où le Système de la cour, 1997, aaO. (FN34) S. 512.

39 Y. Coirault, L'optique de Saint-Simon, Paris 1965, S. 432, Anm. 61.

40 Le Roy Ladurie, aaO. (FN36) S. 53.

41 Saint-Simon, aaO. (FN27) S. 1013.

42 Madeleine Foisil, L'enfant Louis XIII. L'éducation d'un roi, 1601-1617, 1996, Avant-Propos, S. 11.

43 Saint-Simon aaO. (FN27) S. 1014. Die im Rahmen einer ins Auge gefassten »bistoire métallique«, von Saint-Simon beigesteuerte »explication historique", einer die Sammlung eröffnenden "médaille commémorative de Louis XIII. « fiel der Eifersucht derjenigen zum Opfer, die vorhersahen, "que le terme des prospérités de son règne fût arrivé, et qu'ils n'auraient désormais à le loner que de sa constance". Vgl. Saint-Simon, Mémoires II, Pléiade, S. 191 und Anm. S. $1259 f$. 
souverain trop méconnues " ${ }^{44}$ - eine Aufhellung durch einen einfühlsamen Beobachter, der noch »sous la Régence même semble être le contemporain de Louis XIII«.45

Bei genauerer, eine Analyse des Journal de Jean Héroard einbeziehender Betrachtung tritt nunmehr eine über dem Durchschnitt liegende Persönlichkeitsstruktur in Erscheinung. ${ }^{46}$ Weder Müßiggänger, noch auf Gelehrsamkeit bedacht verkörpert Ludwig »une haute idée des vertus et de l'exercise du métier du roi. Mais surtout, Louis XIII a appris par la pratique et l'éprenve, plus que nul autre avant lui et après lui«, ${ }^{47}$ eine am Tatsächlichen erprobte Ertüchtigung: »Cela s'apprend, so Pierre Chaunu, sur le tas, non sur les bancs de l'école ${ }^{48}$ Allerdings kommt Foisil nicht umhin insbesondere dem seit 1624 amtierenden Kardinalpremier ein weltanschaulich fundiertes, auf Durchsetzungskraft angelegtes Politikverständnis zuzusprechen: »la puissance de la réflexion politique sera apporté lorsque à partir de 1624, il sera devenu le principal ministre.$^{49}$ Sie findet ihren erkennbaren Niederschlag im Politischen Testament ${ }^{50}$ und in einem von Leibniz herangezogenen, neuerlich wiederentdeckten Fallbeispiel.

Enttäuscht über bislang Unterbliebenes, unterzieht sich Saint-Simon nun selbst dieser Aufgabe, "mais sous la plus scrupuleuse direction de la vérité la plus exacte.${ }^{51}$ Gleich eingangs blickt der Autor auf die dem pädagogischen Anliegen der »Constantia" verpflichtete école de l'adversité. Sie beflügelte die Widerstandskraft des ersten Bourbonen, "l'endurcit aux fatigues $d u$ corps et de l'esprit, aux contretemps, aux désastres «. ${ }^{52}$

Die darüberhinaus von Saint-Simon als ausschlaggebend erachteten Erziehungsschwerpunkte haben ihren Ausgangsort in leitbildartig geformten Anschauungen. So übertrug Jeanne d'Albret, vertueuse, courageuse und sich durch Lebenstüchtigkeit auszeichnend, als Mutter des Thronfolgers und künftigen Königs Henri IV. seine Unterweisung den besten Ratgebern »qui lui apprirent, tout ce que devait savoir un prince qui

44 Faugère aaO. (FN35) Avant propos III.

45 Faugère aaO. (FN35) S. XV. Eine Tendenz "au panégyrique" erkennt Fabre de Navacelle, Saint-Simon: Parallèle de trois premiers rois Bourbons, in: L'investigateur, 51, 1880, S. 322.

46 Par le regard aigu qu'il (Héroard) a porté sur l'enfant, il a saisi ses virtualités profondes et nous a fait percevoir le devenir que sa vie d'adulte devait confirmer. Le goût des physiques et des jeux de la guerre, c'est le roi soldat; les dons pour la musique et le dessin, c'est le roi compositeurinterprète et artiste; la répugnance à l'exercice intellectuel, c'est la préférence du roi pour l'action. Siehe Journal de Jean Héroard. Tome premier sous la direction de Madelaine Foisil, Paris 1989, S. 348.

47 M. Foisil, L'enfant Louis XIII, aaO. (FN44) avant propos S. 16.

48 Zitiert bei M. Foisil, aaO. (FN44). aaO. S. 16. Siehe dazu: Isabelle Flandrois, »Linstitution du prince au début du XVIIe siècle, Paris 1992, Conclusion, S. 218.

49 M. Foisil, aaO. S. 16. Siehe dazu: Isabelle Flandrois, aaO.

50 Carl Siedschlag, »Die Rezeption des politischen Neustoizismus im politischen Testament Richelieus « in: Zeitschrift für Politik, 3, 2008, S. 315f.

51 Saint-Simon, Parallèle, aaO. (FN27), S. 1014.

52 Saint-Simon, aaO. (FN27). Parquoy vous qui estes encor ieune si vous me croyez, so ermahnt Langius seinen Gast, vous demeurerez stable et vous affermerez contre la douleur qui vous fait la guerre: or avez vous surtout besoin de Constance: car quelques uns ont vaincu en combatant mais iamais en fuyant...(I, 3f.) In: Juste Lipsius de la Constance, Paris 1609, S. $7 \mathrm{ff}$. 
avait besoin du tout... qui ne pouvait prospérer qu'à force de courage, de suite et d'industrie, et qui devait lutter sans cesse contre les tempêtes $d u$ debors et du dedans «. ${ }^{53}$

Dem Dauphin widerfuhr dagegen nicht Vergleichbares. Vom Hofe verbannt - »c'était le tirer de prison pour le faire monter sur le trône " ${ }^{4}$ - so ergeht ein zugespitztes, erkennbare Vorbehalte gegenüber der Regentin Marie de Médicis begründendes Urteil.

In einer ersten Zwischenbilanz hat sich für Saint-Simon gezeigt, dass bei unterschiedlicher Persönlichkeitsstruktur von Sohn und Enkel Heinrichs IV. ihre Früherziehung übereinstimmenden Ansichten ihrer Mütter folgte: »Louis XIII et Louis XIV eurent donc pour l'ignorance et la retenue une éducation à peu près semblable «. ${ }^{55}$ Davon hebt sich überdeutlich, geradezu modellhaft diejenige Heinrichs IV. ab: »Tout y contribua à le former au monde, aux troupes, à la politique, à la guerre, à lui aiguiser l'esprit et le courage. $" 56$

Rücken famille et domestique in ein sich ausweitendes Blickfeld, so erfährt bislang Beobachtetes eine Verstetigung. Dem Selbstbehauptungswillen des ersten Bourbonen stand nichts im Wege, »tous les grands avantages quipeuvent former un béros furent donc uniquement $d$ u côté d'Henri $I V$ «. ${ }^{57}$ Im Enkel kumuliert alles seinen Aufstieg zum »roi soleil Befördernde. ${ }^{58}$ Das dagegen dem Vater auferlegte Los sucht jedoch seinesgleichen, "Tout ce qui peut abrutir et accabler un jeune prince ... fut le partage de Louis XIII «. ${ }^{9}$

Guerre et guerrier stellen auch für Saint-Simon eine unumgängliche Bewährungsprobe dar. Denn es ist das den Leidenschaften innewohnende Verhängnis, dass dem Bedürfnis Vorschub leistet, den Weg der Gewalt einzuschlagen. Allein ein den Geboten der prudence militaire verpflichteter Kriegsherr ${ }^{60}$ ist imstande das Übel zu meistern. »La valeur, et l'art de savoir faire la guerre est donc nécessairement devenu un point capital chez tous les peuples. $" 61$

53 Saint-Simon, aaO. (FN27) S. 116.

54 Saint-Simon, aaO. (FN27) S. 1018.

55 Saint-Simon, aaO. (FN27) S. 1023.

56 Saint-Simon, aaO. (FN27) S. 1024.

57 Saint-Simon, aaO. (FN27) S. 1031. Siehe dazu Jacques Hennequin, Henri IV dans ses oraisons funèbres ou la naissance d'une légende, 1977. "Le Héros guerrier«, S. 145f.: Der "prédicateur Fenouillet «(aaO. S. 157) lobt »ses vertus de vigilence, prévoyance, constance, prudence, adresse, promptitude, courage et son esprit toujours victorieux posé au dessus de la fortune et du malheur . Zur "vertu de jugement dans le choix des hommes" bemerkt Fenouillet (aaO. S. 163): "par un grand ingement, il faisait le choix de ses serviteurs pour les employer selon leur capacité". Für Binet (aaO. S. 158) hat der König, "le plus grand chef de guerre qu'ayent iamais veu les armées "(Coeffeteau) als "vir totus ex sapientia et virtutibus factus" zu gelten. Der Tenor der Lobpreisungen ist also auch an dieser Stelle eindeutig.

58 Saint-Simon, aaO. (FN27) S. 1029.

59 Saint-Simon, aaO. (FN27) S. 1031.

60 Vgl. Lipsius, Politiques aaO. (FN8) V, 2, S. 158. "Car comment se pourroit il autrement conserver luy, ses biens et les siens? Une simple force et toute nue serviroit peu pour cela, si elle n'estoit modérée par quelque art et conseil, c'est à dire par une Prudence militaire.".

61 Saint-Simon, aaO. (FN27) S. 1031; vgl. Lipsius, Politiques, aaO. (FN8) V, 2. Je maintien donc que la prudence militaire est sur toutes choses nécessaire au Prince, tellement que sans icelle à peine pourroit on dire qu'il serait Prince. 
Es mehrt den Ruhm eines Monarchen, so Saint-Simon zusammenfassend, der das sagesse, équité, modération einschließende grand talent militaire und das talent politique in seiner Person vereinigt, sich nach außen Respekt verschafft, im Inneren - als "père et les délices de ses peuples" - seiner landesherrlichen Verantwortung gerecht wird. ${ }^{62}$

Die Heinrich IV. zuerkannten vertus militaires ${ }^{63}$ machen ihn kenntlich als einen in Kriegsdingen kundigen und vielfach erprobten Heerführer, der sich durch Tapferkeit und eine in der école d'adversité gestählte, unbezwingbare Widerstandskraft auszeichnete. Aber auch dem Sohn bleibt es nicht versagt, par ses seules forces personnelles seine Ebenbürtigkeit unter Beweis zu stellen »et montrer à l'Europe un homme, un roi, un capitaine ${ }^{64}$

Das unerwartet Eingetretene fordert den Vergleich heraus. Schon die erfolgreiche Belagerung von La Rochelle führt valeur und capacité des zweiten Bourbonen vor Augen, ${ }^{65}$ die in ihrer Einzigartigkeit im Begriff ist, die supériorité des Vaters zu übertreffen. Aber die Erfolge auch künftiger exploits gewinnen ihren besonderen Glanz durch die "fermeté inaltérable de sa volonté, de sa vigilence, de sa patience", durch seine Durchsetzungskraft gegenüber einer "opposition constante de tous ses généraux, de tous ses ministres, du cardinal de Richelieu " ${ }^{66}$ Schließlich ist Ausweis soldatischer Tugend eines vorbildlichen Heerführers der persönliche Einsatz des Königs, der unerschrocken "montre à ses soldats un soldat tout pareil à eux, qui combat en personne au milieu $d^{\prime}$ 'eux ${ }^{67}{ }^{67}$ So ist zu fragen, ob die bei Vater und Sohn beobachtete Wertschätzung der zum Maßstab vornehmster Herrscherpflichten erkorenen »vertu militaire - ceste imperiale estoit la propre d'un bon chef ${ }^{68}$ sich auch beim Enkel zu erkennen gibt.

Der sich nach dem Tod Mazarins (1661) auftuende Handlungsspielraum ${ }^{69}$ führt sogleich zu mehreren actions militaires. Damit einhergehend steigert sich das Bedürfnis nach intensiver militärischer Unterweisung, eine Aufgabe die Monsieur le Prince et Monsieur de Turenne oblag, »ces deux premiers capitaines de ce siècle « ${ }^{70}$

Der vergleichende Blick auf den aufstrebenden dritten Bourbonen scheint auf eine Akzentverschiebung hinzudeuten: vertu militaire wird gleichsam zum Inbegriff einer ungezügelten militant ${ }^{71} \mathrm{zu}$ verwirklichenden Expansionsstrategie. ${ }^{72} \mathrm{Im}$ Übrigen, so

62 Saint-Simon, aaO. (FN27) S. 1032.

63 Saint-Simon, aaO. (FN27) S. 1032 f.

64 Saint-Simon, aaO. (FN27) S. 1035.

65 Saint-Simon, aaO. (FN27) S. 1048.

66 Saint-Simon, aaO. (FN27) S. 1050.

67 Saint-Simon, aaO. (FN27) S. 1051. Entsprechendes bei Lipsius aaO. (FN8) V, 14, S. 200f. »Des chefs d'armée. La présence du prince augmente la force et le courage à l'homme de guerre.".

68 Lipsius, aaO. (FN8) V, 2, S. 158.

69 Saint-Simon, aaO. (FN27) S. 1051.

70 Saint-Simon, aaO. (FN27) S. 1052.

71 Zum Gewicht der die réorientation voulue par le souverain verkörpernden, sich zu den valeurs dominantes à l'époque bekennenden noblesse militaire, deren Ansehen sich durch besondere Hingabe auszeichnet und verdientermaßen ihre Belohnung erfuhr, siehe Jean-Christian Petitfils, Louis XIV, 1997, S. 333. Vgl. dazu Lipsius, aaO. (FN8), V, 8, S. 198.

72 Christian Petitfils, aaO. (FN69) S.319ff., macht auf eine zunehmend objektivere Betrachtungsweise der »diplomatie agressive et conquérante« aufmerksam. Darüberhinaus bleibt die 
meint Saint-Simon beobachtet zu haben, ähnelt die anlässlich seiner exploits personnels angewandte Vorgehensweise derjenigen seiner Vorgänger auch mit Blick auf ein ihn gleichermaßen auszeichnendes Charakterprofil: "Le roi reste stoique face à la douleur physique, aux denils et aux défaites « ${ }^{73}$ An diesem Punkt angelangt, lässt sich für den kritischen Betrachter die Schwierigkeit nicht verhehlen, den den augustes têtes geschuldeten Respekt mit dem Auffinden der Wahrheit in Einklang zu bringen. Beiden Ansprüchen genüge zu tun, nötigt also einen mittleren Weg einzuschlagen. ${ }^{74}$

Jenseits des Militärischen breitet Saint-Simon ein die Charaktereigenschaften erhellendes Sittenpanorama aus - les moeurs - und wird sie in ihrer Schlüsselfunktion einem nach eigenen Leitvorstellungen zu bemessenden Tauglichkeitstest unterziehen. Seine ungezügelte Leidenschaft gerät Heinrich IV. zum Verhängnis, "fut son plusfuneste écueil, le malbeur de sa vie " ${ }^{75}$ so lautet das Urteil, das sogleich durch die in Augenschein genommenen amours éclatants seine Bestätigung findet. In sklavischer Liebe der schönen Corisande d'Andoins verfallen, erscheint die Pflichtvergessenheit eines im gereiften Mannesalter stehenden Heerführers würdelos. Gabrielle d'Estrées - ensuite la passion d'Henri IV - geriet zum Anlass für eine von Saint-Simon als abstoßend empfundenes Scheidungsdrama.

Ein vereitelter Hochverrat machte den Plan der Marquise de Verneuil zunichte Marie de Médici den Platz einer concubine anzuweisen und den rechtmäßigen Thronfolger zum bâtard zu degradieren. »C'est ici«, so fasst Saint-Simon die skandalträchtigen Vorgänge zusammen, »où la faiblesse d'Henri IV paraît jusqu'à l'incroyable «. ${ }^{76}$ Es bleiben jedoch Fragen. Lässt sich ein solcherart Manko mit seinen fatalen Begleiterscheinungen vereinbaren, "avec un courage d'esprit et de coeur " in ein und derselben Person? Und wer erschrickt nicht vor den unsäglichen »effets de l'amour, ce cancer dévorant "wenn es ein Monarch ist, der ihnen zum Opfer fällt. ${ }^{77}$

Ihm zur Seite stand jedoch der surintendant Sully, der Schlimmeres zu verhüten trachtete durch franchise und fermeté avec Henri IV. ${ }^{78}$ Gesellen sich noch hervorstechende Charaktermängel hinzu, so bleibt nur Raum für eine ernüchternde Bilanz: »il est vrai que dans les plus grands hommes, l'bumanité perce partout «. ${ }^{79}$

Der für Mademoiselle de Hautefort, "fille d'honneur de la Reine" empfundenen leidenschaftlichen Zuneigung nachzugeben erscheint dem Sohn (Ludwig XIII.) unstatt-

Frage nach dem Zusammenhang von »prudence militaire « und dem spektakulären »effort de renouvellement « - »l'armée est la seule force organisée dans ce grand Etat sous-administré« (aaO. S. 344) - unbeantwortet.

73 Olivier Chaline, Le règne de Louis XIV, 2005, S. 96.

74 Saint-Simon, aaO. (FN27) S. 1055.

75 Saint-Simon, aaO. (FN27) S. 1056. Zur Verbindung von ausschweifenden Sinnesfreuden mit Volkstümlichkeit siehe Ernst Jünger, Siebzig verweht, IV, Stuttgart 1995, S. 309.

76 Saint-Simon, aaO. (FN27) S. 1061. Mme de Simier, fille d'honneur de Catherine de Médici, bemerkte einmal, sich an den Vorgänger erinnernd, beim Anblick Heinrich des IV.: J'ay veû le Roy, mais je n'ay pas veû Sa Majesté. Siehe Tallemant des Réaux, Historiettes I, 1960 (Pléiade), S. 15 u. $40 f$.

77 Saint-Simon, aaO. (FN27) S. 1063.

78 Saint-Simon, aaO. (FN27) S. 1063.

79 Saint-Simon, aaO. (FN27) S. 1064. 
haft, ${ }^{80}$ weil »Dieu me le défend «. Ihm zu gehorchen ist oberste Herrschertugend. So gilt es die Mühen der Selbstüberwindung auf sich zu nehmen "pour me surmonter moimême«, gepaart mit Wachsamkeit gegenüber den Fallstricken der Verführung - »les occasions et les humanités".

Der Appell an den Standhaftigkeit gewährenden »robur animi« bleibt also unüberhörbar: "je dois être contre le crime et le scandale, et demeurer le maitre de moimême " ${ }^{81}$ Zur "pureté des moeurs sans tache" tritt die Bescheidenheit hinzu. »Une modestie qui accompagne toutes ses actions ${ }^{82}$ Sie gestattete ihm erworbene Verdienste neidlos anzuerkennen, eigene herausragende Leistungen dem Kardinalpremier gutzuschreiben und damit dessen Ruhm gemehrt zu haben. Das Bewusstsein für sein ihm aufgetragenes Tun rechenschaftspflichtig zu sein, erfüllte ihn mit gottesfürchtiger Demut (humilité). Sie war fest verwurzelt in einer "solide et parfaite piété ${ }^{83}$ Sie trat vielfältig in Erscheinung: durch Hilfsbereitschaft, maßvolle Freigiebigkeit, durch geduldige Hinnahme aller ihm begegnenden Widrigkeiten - ein Verhalten, dass ihm wie Saint-Simon anmerkt, zu unrecht als Schwäche angelastet wird, "d'être gouverné même malgré lui ${ }^{84} \mathrm{Zu}$ den ihn auszeichnenden Tugenden zählt der Autor eine maßhaltende Lebensführung, "la sobriété", die Wahrung der "bienséances de son état royal «, seinen Gerechtigkeitssinn, der ihm den "saint et rare titre de Juste" eintrug, seine Bereitschaft das Verdienstvolle zu würdigen und seine Abscheu gegenüber allem Undurchsichtigen, »il voulut l'ordre et la règle partout".

Auch den Sohn, Ludwig XIV., wird Saint-Simon einer solchen das neustoisch-weltanschauliche Empfinden abbildenden Betrachtungsweise unterziehen. Dabei übersieht der nüchtern-skeptische Blick auf das Unzulängliche nicht seine "grandes qualités" angesichts im letzten Lebensjahrzehnt erlittener persönlicher Schicksalsschläge und militärischer Niederlagen: »quelle distance", so muss Saint-Simon eingestehen »de ces temps si longuement florissants ${ }^{85}$ Aber Ludwig lässt sich nach außen hin von den sich auftürmenden adversités nicht überwaltigen. "Si sa fermeté dans la suite des divers malheurs si accablants de ses dernières années a paru héroique, la piété, l'bumilité, la soumission avec lesquelles il les a reçus comme venant de la main de Dieu, ne l'ont pas été moins "86 - so reiht er sich ein in den Kreis der kämpferisch Standhaften.

In einem "court retour sur les trois monarques « verbirgt Saint-Simon abermals nicht seine Bewunderung, die er der Persönlichkeit Ludwigs III. entgegenbringt »sa valeur, sa capacité militaire, son esprit et sa rare et constante piété «, seiner bis an die Grenzen der Selbstverleugnung reichenden Bescheidenheit, die der Pflichterfüllung jedoch keine Ein-

80 Saint-Simon, aaO. (FN27) S. 1064. Einen die Selbstdisziplin einfordernden Ratschlag hatte bereits Lipsius erteilt: Soyez chaste. Il ne peut avoir rien d'honeste en une ame corrompue de paillardise. Politiques, aaO. (FN8), II, 17, S. 47.

81 Saint-Simon, aaO. (FN27) S. 1065.

82 Siehe dazu Lipsius, aaO. (FN8) Politiques II, 7, S. 45f.

83 Saint-Simon, aaO. (FN27) S. 1067. Siehe dazu Lipsius, Politiques, aaO. (FN8) I, 3, S. 5.

84 Saint-Simon, aaO. (FN27) S. 1027.

85 Saint-Simon, aaO. (FN27) S. 1079.

86 Saint-Simon, aaO. (FN27) S. $1097 f$.

ZfP 59. Jg. 4/2012 
bußen gestattet ${ }^{87}$ "travaillant sans cesse de corps et d'esprit dans l'état de monarque où Dieu l'avait fait naître ${ }^{88}$

Nachhaltige Aufmerksamkeit schenkt Saint-Simon der Bewältigung des Herrschaftsauftrages - »le gouvernement«. Lob erfährt Heinrich IV. für eine »mérite« und »capacité " achtende, dabei das konfessionelle Konfliktpotential neutralisierende Auswahl seiner Ratgeber, "pour puiser des lumières ${ }^{89}$ - eine nötige Hilfestellung "pour savoir se conduire lui-même dans l'infinie diversité des choses, des conjonctures et de leurs complications ${ }^{90}$ So ermöglicht schließlich die Zusammenschau ein eindeutiges Urteil: »C'est ainsi qu'un sage roi gouverne en effet et sait s'empêcher d'être gouverné. "91

Das von Vater und Sohn erworbene Ansehen und ein herausragender Kardinalpremier lassen dagegen die Regierungstätigkeit Ludwig XIII. wenig glanzvoll erscheinen, wofür ein hoch angesiedeltes sittliches Anspruchsniveau als mitverantwortlich zu gelten hat: "cette bumilité si vraie et si unique dans un grand roi, et ce détachement de soi-même d'autant plus héroique qu'il fut toujours égal et parfait. «92

Mit dem Tod des connétable de Luynes (1621) setzt die selbstverantwortliche Regierungstätigkeit des jungen Ludwig ein. Er erkennt sogleich, dass ihre erfolgversprechende Bewältigung der Unterstützung bedarf. Denn auch Könige sind Menschen: »Leur tête ni leur temps ne peut suffire à tout. Ils ne peuvent donc se passer de ministres... qui les aident dans les diverses partis du gouvernement et dans des cas singuliers «. ${ }^{93}$ Sich der "capacité de Richelieu " als Premierminister zu bedienen, zeugt für Saint-Simon von einem ausgeprägten Urteilsvermögen - »ce don de discernement «. Seine getroffene Wahl »ne pouvait être meilleur, ${ }^{94}$ une des plus fortes, des plus grandes et des plus heureuses actions de Louis $X I I{ }{ }^{95}$ - gleichsam eine lehrbuchgetreue, dem Ratschlag des Lipsius folgende Vorgehensweise. "Il faut donc, de l'ayde et de l'assistance, et ie croy que le sommaire de la Prudence Royale gist à choisir des hommes prudents. "96

Der vorliegende Befund nötigt Saint-Simon ein sich aus Unwissenheit speisendes Vorurteil zu entkräften, dass der Kardinalpremier sein Ansehen einem schwachen Souverän zu verdanken hat. Ohne das "puissant génie du cardinal de Richelieu «" zu unterschätzen, ist es unbestreitbar, dass alle großen Unternehmungen erst nach gemeinsamen, geheimen Beratungen in Angriff genommen wurden. Es geschah aber nur mit seinem

87 Saint-Simon, aaO. (FN27) S. $1097 f$.

88 Saint-Simon, aaO. (FN27) S. 1092.

89 Saint-Simon, aaO. (FN27) S. 1099.

90 Eine besondere Würdigung erfährt das Edit de Nantes, ce fut donc le chef-d'oeuvre de la sagesse de la connaissance et de la patience d'Henri IV d'être venu à bout d'une affaire si peu possible, aaO. 1104.

91 Saint-Simon, aaO. (FN27) S. 1110.

92 Saint-Simon, aaO. (FN27) S. 1120.

93 Saint-Simon, aaO. (FN27) S. 1127.

94 Saint-Simon, aaO. (FN27) S. 1122.

95 Saint-Simon, aaO. (FN27) S. 1127.

96 Lipsius, Politiques III, 3, (FN8) S. 52f.

97 Saint-Simon, aaO. (FN27) S. 1129. Ebenso F. Hildesheimer, Richelieu, aaO. (FN 3) S. 302. Der topos littéraire eines roi fantoche, devenu »le valet d'un prêtre« ist mit dem Tatsächlichen unvereinbar. 
Einverständnis, "sa volonté, son concours de roi et de maître «, ${ }^{98}$ ohne dem sich zugleich mehrenden Prestige seines Mitstreiters Abbruch zu tun: "Quel comble de gloire pour Louis XIII de la savoir également mériter et mépriser, et que cette sorte de gloire est héroique et unique «!9 Für Saint-Simon nimmt das Erscheinungsbild Gestalt an eines im guten Sinne denkwürdigen Repräsentanten moralisch-ethisch fundierter, frühneuzeitlicher Herrschaftslehren. ${ }^{100}$

Bis 1661 eher ein "monarque en peinture", genoss Ludwig XIV., nach dem Tod Mazarins, den Vorzug ein glänzendes Erbe anzutreten: »Un royaume paisible et florissant ", hervorragende Minister, erfahrene Diplomaten, hochangesehene Heerführer und im Innern eine kaum noch spürbare Opposition. Ein weites Feld tut sich auf, aber der eingeschlagene Pfad wird von Saint-Simon nicht verlassen: "je me suis proposé d'un parallèle, et non pas d'une histoire ${ }^{101}$

Er richtet seinen Blick auf die zur Hilfestellung bereitstehenden Ratgeber, die ministres en place à la mort du cardinal Mazarin. Das dabei Vorgefundene verkehrt jedoch das lehrbuchmäßig Geforderte in sein Gegenteil. Nicht zuletzt der König selbst bot dafür die Handhabe. Die mit Sach- und Erfahrungswissen ausgestatteten Minister Colbert, Le Tellier, Louvois, ces têtes fortes, hoben ihre Bedeutsamkeit hervor mit einer von SaintSimon missfällig beobachteten »apparence de grandeur des rois asiatiques «: ${ }^{102}$ Sie boten ein vielgestaltiges, Gegensätzliches miteinander verknüpfendes Erscheinungsbild. »Peu d'esprit naturel avec un sens droit, une ignorance générale jusqu'à l'incroyable, de la défiance sur tous gens et choses, une soif de grandeur, d'autorité, de gloire, aber auch Anzeichen von bonté und équité naturelle. " ${ }^{103}$ So wurde er zum Spielball von Ratgebern, die es in Verkennung ihres eigentlichen Auftrages sich angelegen sein ließen, persönlichen Ambitionen den Vorrang einzuräumen. "Comme le petit lui était fort homogène, il s'y attacha avec avidité, en prit titre de se persuader qu'il se gouvernait seul... tandis que le grand, le vaste, les détails les plus importants demeuraient entre leurs mains. «104

Die friedlichen Absichten Colberts und die dabei erzielten Erfolge rufen die Militärs Le Tellier, Louvois auf den Plan. Mit dem Friedensabschluss von Nimwegen gingen sodann die Eroberungspläne eines jungen Monarchen "affamé d'acquérir de la gloire" erstmals in Erfüllung. An dieser Stelle zögert Saint-Simon nicht, die herausragende Befähigung von Louvois hervorzuheben als »un des plus grands ministres «. ${ }^{105}$ Denn er verschaffte den auf großen Widerhall stoßenden, sachlich gebotenen Leitsätzen einer frühmodernen Heeresverfassung nachhaltig Geltung.

98 Saint-Simon, aaO. (FN27) S. 1129.

99 Saint-Simon, aaO. (FN27) S. 1130.

100 Als Beispiel moralischen Fehlverhaltens tadelt Saint-Simon, aaO. (FN27) S. 1165ff. die Illoyalität des für seine Gelehrsamkeit gerühmten Hofbibliothekars Auguste de Thou.

101 Saint-Simon, aaO. (FN27) S. 1174.

102 Saint-Simon, aaO. (FN27) S. 1196.

103 Saint-Simon, aaO. (FN27) S. 11.

104 O. Chaline, Le règne de Louis XIV, 2005, S. 98f. weist eine u.a. auch bei Saint-Simon anzutreffende Fehleinschätzung zurück.

105 Saint-Simon, aaO. (FN27) S. 1177.

ZfP 59. Jg. 4/2012 
Einen hohen Stellenwert beanspruchen ein der Autorität des Heerführers zuträgliches Detailwissen, die Wiederaufrichtung der Militärdisziplin, ${ }^{106}$ »subsistance, service « ${ }^{107}$ ein gesicherter Kenntnisstand bezüglich der Truppenteile und ihres Führungspersonals, »la connaissance intérieure des corps et de leurs officiers «. ${ }^{108}$ Aber mangelt es an einem ebenbürtigen Gegenüber, gewinnt die Eigensucht die Oberhand: »senl, libre et volant de ses ailes sous un roi qu'il avait su offusquer par la gloire, infiniment pernicieux en ce qu'il se compta pour tout et l'Etat pour rien «. ${ }^{109}$

So gibt sich Louvois zu erkennen als ein grand capitaine, der von einer zügellosen Kriegsleidenschaft befallen, die Aufhebung des Toleranzediktes als Chance für eine weitere Bewährungsprobe ansieht und dabei das Vorgegebene in sein Gegenteil verkehrt. ${ }^{110}$

Ein Einspruch gegen sein militärisches Vorgehen "qu'est ce qu'il désirait le plus ardemment « ${ }^{111}$ stand für Louvois nicht zu erwarten, denn der König, von missionarischem Eifer beherrscht »se croyait un apôtre". So kommt Saint-Simon nicht umhin die durch den einsetzenden religiösen Verfolgungsterror verursachten Folgen zu beklagen, weil unvereinbar mit christlicher Nächstenliebe, "qui est l'âme de la religion. La révocation de l'Edit de Nantes, sans le plus léger prétexte et sans aucun besoin... déchira les familles, arma parents contre parents pour avoir leur bien et les laisser mourir de faim ... «. ${ }^{112}$

Solcherart bereits von Lipsius gesammelte Erfahrungen sind nur ein Glied in einer Kette allzeit wahrnehmbarer Bedrängnisse. An den Einzelnen richtet sich deshalb die Aufforderung, durch eine von kampfentschlossener "philosophia practica" erwirkte stoisch-männliche Haltung - »robur animi«-den unvermeidlichen Heimsuchungen standhaft zu begegnen. ${ }^{113}$

Zwei Maximen, die Ludwig XIV. nach dem Tode Mazarins seiner innen- und militärpolitischen Vorgehensweise zugrunde legt, misst Saint-Simon eine besondere Bedeutung bei: »l'une, excellente, de ne vouloir plus de premier ministre «, keine Beteiligung von »ecclésiastiques« an den Regierungsgeschäften. Eine zweite Vorgabe hält Saint-Simon, aus eigenem Standesinteresse heraus betrachtet, für nicht vertretbar: »ce fut de ne mettre jamais dans le conseil que des gens de fort peu«. Es schlug die Stunde der Emporkömm-

106 Vgl. Lipsius aaO. (FN 8), V, 8, S. 174. Chaline, Le règne de Louis XIV,(FN 102), unterstreicht "la volonté farouchement incarnée par Le Tellier, puis surtout par Louvois" das traditionell auf Unabhängigkeit bedachte Offizierscorps einer discipline plus rigide zu unterwerfen "assortie d'une formation professionelle plus systématique". Vgl. dazu Lipsius aaO. (FN 8), V, 15, S. 202.

107 Vgl. Lipsius aaO. (FN 8), V, 6, S. $168 \mathrm{ff}$.

108 Vgl. Lipsius aaO. (FN 8), V, 7, S. 171.

109 Saint-Simon, aaO. (FN27) S. $1177 \mathrm{f}$.

110 »Il n'est pas question seulement", so gibt Lipsius, Politiques, aaO. (FN 8), V, 15, S. 203f., zu bedenken, "de rechercher en un souverain et parfait chef d'armée, l'habilité de scavoir faire bien la guerre, il y a beaucoup d'autres excellentes parties, qui sont comme compagnes et ministres de ceste vertu«.

111 Saint-Simon, aaO. (FN27) S. 1180.

112 Saint-Simon, aaO. (FN27) S. 1181.

113 Justus Lipsius, Von der Bestendigkeit (De Constantia), hrsg. Von Leonard Forster, Stuttgart 1965, S. 128. 
linge, die den Gunstbezeugungen des Königs ausgeliefert, alles daran setzen »à bien servir et à lui plaire«. ${ }^{114}$

So prangert Saint-Simon unverblümt die auf die Herausgehobenheit des Monarchen fixierte, zu Schmeichlertum und Willfährigkeit ermunternde Gesinnung an: »il ne se plut jamais que dans le tremblement devant lui et dans l'aveu d'ignorance «. ${ }^{115}$ Eine derart abschätzige Verfälschung eines auf hilfreichen Ratschlag abzielenden Klugheitsgebotes war unvereinbar mit der römisch-stoischen Pflichtenlehre, die besondere Aufnahme fand bei den eine »ecclesia militans « (Oestreich) bildenden Trägern des neuzeitlichen Machtstaates: Fürstentum, Beamtentum und Heer. ${ }^{116}$

Dem neuen Hilfspersonal, für Saint-Simon mit dem Makel der Unebenbürtigkeit behaftet, fällt es unter den gegebenen Umständen leicht »de s'élever sur les ruines des seigneurs jusqu'à les fouler aux pieds«. Hierzu leistete der für einen Modernisierungsschub verantwortliche Heeresreformer Louvois einen revolutionär anmutenden Beitrag indem es ihm gelangt "à rendre toute seigneurie et toute noblesse peuple, dont la nécessité $d u$ service militaire lui fournit les moyens «. ${ }^{117}$

Die von Louvois auf breiter Front in Gang gesetzte militärische Unterweisung erfüllt für Saint-Simon in Wirklichkeit den Zweck althergebrachte Befehlsstrukturen aufzubrechen, um alle Militärangehörigen auf gleicher Stufe anzusiedeln, »les plus grands seigneurs se trouvaient encore confondus avec les soldats de fortune et ce qui était encore pis ... avec des gens de peu, quelquefois de rien " ${ }^{118}$ denen durch fragwürdige Machenschaften der Offiziersrang zugesprochen wurde. »Telle fut la plaie de toute la noblesse et de la plus haute." Droht somit spezifischen Belangen Gefahr, so vermag der 1702 wegen zu langsamer Beförderung aus dem Militärdienst ausgeschiedene Duc seine Befangenheit nicht zu verbergen gegenüber den von der "prudence militaire" ausgehenden Reformanstößen, die das disziplinierte Berufsheer in den Vordergrund rückten, die, als »formidable Armee«, nicht eigentlich dem Bedürfnis der Landesverteidigung, sondern den Machtbestrebungen der Krone diente. ${ }^{119}$

Um mit Blick auf militärische Aufstiegschancen eine "parfaite égalité" zu erzielen, setzt Louvois nunmehr auf "ancienneté " beruhende Beförderungsrichtlinien in Kraft, eine von Saint-Simon als schädlich bewertete Maßnahme, durch die dienstliches Desinteresse gegenüber »émulation, application, toute envie de s'instruire» die Oberhand ge-

114 Saint-Simon, aaO. (FN27) S. 1187.

115 Saint-Simon, aaO. (FN27) S. 1188.

116 J'ay toujours remarqué, so Lipsius, Politiques, III, 3, S. 53, que tant les bons conseils ont eu lieu, les Royaumes, les peuples, les villes ont heureusement conservé leur authorité.

117 Dieses Urteil relativiert Le Roy Ladurie, Saint-Simon ou le système de la cour, 1997, S. 83. On naît brave ou lâche à la guerre comme on vient au monde avec du sang bleu; personne n'y peut rien.

118 Saint-Simon, aaO. (FN27) S. 1188.

119 O. Hintze, Gesammelte Abhandlungen III, Regierung und Verwaltung, 1967, S. 382. Zitiert bei G. Oestreich, Zur Heeresverfassung der deutschen Territorien, aaO. (FN 14) S. 310.

ZfP 59. Jg. 4/2012 
winnt. ${ }^{120}$ Die Erfahrung gebietet demgegenüber eine praxisorientierte Vorgehensweise, ${ }^{121}$ die den über militärische Autorität verfügenden Vorgesetzten, die Aufgabe überlässt, einschlägig qualifizierte Nachwuchskräfte auszuwählen. ${ }^{122}$ Aber ein Verlassen des eingeschlagenen Irrwegs steht nicht zu erwarten. Der auf den Erhalt seiner Machtfülle bedachte Louvois "a éteint dans les généraux et les particuliers toute émulation, toute instruction, et a tari en France les capitaines, sans nulle espérance d'en voir plus se former et s'élever «. 123

Nach den für Ludwig unbefriedigenden, von außenpolitischem Prestigeverlust begleiteten Utrechter Ergebnis gebietet es die mit machtpolitischen Zielsetzungen übereinstimmende patriotische Pflicht des sich als »vrai Français» begreifenden Saint-Simon Partei zu ergreifen "quand la vérité n'arrête point ses louanges " ${ }^{124}$ Es ist die aufscheinende innere Größe des Königs, die dem ihm zuerkannten »nom de Grand justement acquis« nun erst seine Berechtigung verleiht. Denn Ludwig, vor außergewöhnliche Herausforderungen gestellt, findet Zugang zu der für Jahrzehnte der geistigen Elite Europas vermittelten stoischen Ethik des Leidener Universalgelehrten Justus Lipsius. ${ }^{125}$

An der Tragweite dieser Annäherung lässt Saint-Simon keinen Zweifel aufkommen: "La grandeur de son âme, sa fermeté, sa stabilité, son égalité, un courage à l'épreuve des plus épouvantables revers et des plus cuisantes peines, une force d'esprit qui ne se cache rien, qui ne se dissimule rien, qui voit les choses comme elles sont; qui de là s'bumilie en secret sous la main de Dieu" 126 - gleichsam die Quintessenz der zum "robur animi" erziehenden Constantia, einer wie Hopscher formuliert, apothéose de la philosophie stoicienne, ${ }^{127}$ deren Lektüre der bekannte Rostocker Theologe David Chytraeus seinen Studenten mit den Worten empfahl: »Kauffets jr Staudenten und lesets, dann in tausent Jharen ist dergleichen Buch in Philosophicis nicht geschrieben und gesehen worden.«128

Weiterführende Betrachtungen gelten nun der comparaison des gouvernements. Heinrich IV., als Heranwachsender durch Erziehung und Zeitumstände begünstigt, war als »capitaine" und eigenverantwortlich Handelnder, den Rat herausragender Mitarbeiter

120 Saint-Simon, aaO. (FN27) S. 1190. Zu den »cinq marques d'un bon chef d'armée« siehe Lipsius, Politiques, V, 15, S. 203. Zur der bei Saint-Simon anzutreffenden "obsession $d u$ rang", siehe O. Colombe, L'ordre morale politique et religieux d'après des Mémoires de Duc de SaintSimon, 1982, S. $87 \mathrm{f}$.

121 "Pour le dernier", so Lipsius, Politiques, V, 15, S. 206, "j'ay requis l'Authorité. Elle est fort propre pour faire la guerre, car ce que les ennemys et ce que les amis et aliez croyent du general et des capitaines importe beaucoup en cela.".

122 Zur Election Siehe Lipsius, Politiques, V, 12, S. $188 \mathrm{f}$.

123 Saint-Simon, aaO. (FN27) S. 1191f.

124 Saint-Simon, aaO. (FN27) S. 1225.

125 Näheres zu Auflagehöhe und Verbreitung bei G. Oestreich, Antiker Geist und moderner Staat bei Justus Lipsius (1547 - 1606), Göttingen 1989, S. $213 f$.

126 Saint-Simon, aaO. (FN27) S. 1266.

127 Sh. Oestreich, Antiker Geist, aaO. (FN 123) S. 19, Anm. 70.

128 Justus Lipsius, Von der Bestendigkeit. Hrsg. Von Leonard Forster, 1965, Vorrede von Viritius $\mathrm{zu}$ seiner Übersetzung von Lipsius. 
nicht verschmähender Monarch, gleichermaßen befähigt und durfte sich glücklich schätzen, »d'être doué d'un esprit nerveux et mâle qui ne se laissait manier ni gouverner «. ${ }^{129}$

Vergleichbares gilt nicht für den Sohn. Umso mehr grenzt es für Saint-Simon an ein Wunder, dass ein in völliger Unkenntnis gehaltener Thronerbe, seiner Fesseln ledig, selbstständig das notwendige, das Kriegswesen einbeziehende Herrschaftswissen erwarb, dass »la valeur de coeur et la volonté extrême qui fait le courage d'esprit« das ihm Zugefügte überwinden half.

Ein Vergleich der Regentschaft der drei Monarchen hat hinsichtlich des Lebensalters und der erforderlichen Befähigung bei Antritt des Herrscheramtes von unterschiedlichen Gegebenheiten auszugehen. Wiederum sticht dabei die ungünstige Ausgangslage des Mittleren hervor, "Louis XIII n'avait pas seize ans lorsque la mort du maréchal d'Ancre le sortit du sépulcre où il avait été enfermé«. ${ }^{130}$

Ein dem Vergleich förderlicher Gesichtspunkt gilt dem angemessenen Einsatz des Militärpotentials. Die Heinrich IV. und seinem Sohn zugesprochenen justes motifs des guerres dienen der Sicherung der Souveränität und der Wahrung der Unabhängigkeit. "Tel fut l'objet constant de leurs armes au-dedans et au-debors, de leurs alliances et de toute la conduite de leur règne. " ${ }^{131}$ Der Enkel dagegen, »séduit par Louvois, voulut être conquérant et s'abandonna à toute la superbe de la gloire «. ${ }^{132}$ So treten hier unter Inkaufnahme vieler Fehlentscheidungen dem ethisch-moralischen Anliegen der prudence militaire abgewandte, von Lipsius verworfene motifs des guerres zutage: "la seule et plus ordinaire cause qu'empoignent comunement la plus part des Roys ... pour faire la guerre, c'est une profonde et extreme ambition de regner et une ardente convoitise des richesses«. 133

Ausgehend vom Wunsch nach Rehabilitation Ludwig XIII. bemisst Saint-Simon als eine Art Zwischenbilanz den Stellenwert einer die jeweilige Herrschaftsausübung beeinträchtigende Einflussnahme und so steht einer ihm stichhaltig erscheinenden Urteilsfindung nichts im Wege: »Henri IV ne fut ni ne parut jamais gouverné. Louis XIII le parut et ne le fut point en effet. Louis XIV le fut toujours et le parut toujours. «134

Das Herrschaftsgebahren des Letzteres, so muss Saint-Simon einräumen, setzt seinem Vorhaben Grenzen. Denn nun fallen Wahrheits- und Gerechtigkeitsliebe einem alles überschattenden Ruhmbedürfnis und grenzenloser Herrschsucht zum Opfer - eine irritierende Wahrnehmung, die keine andere Wahl zulässt: »N'entreprenons donc point de

129 Saint-Simon, aaO. (FN27) S. 1233.

130 Saint-Simon, aaO. (FN27) S. 1243.

131 Saint-Simon, aaO. (FN27) S. 1244. Vgl. Lipsius, Politiques, V, 3, S. 162: »Il y a une double cause de iuste guerre: la Deffensive et l'Offensive. Elle (la Deffensive) n'est pas seulement iuste, mais aussi necessaire, veu que en icelle la force n'est que par la force seule empeschée.«.

132 Saint-Simon, aaO. (FN27) S. 1244. J. Cornette, Le roi de guerre, aaO. (FN 4) S. $261 \mathrm{ff}$.

133 Lipsius, Politiques, V, 3, S. 160.

134 Saint-Simon, aaO. (FN27) S. 1255. Zum Einfluss des Karnidalpremiers behält zuvor Gesagtes seine Gültigkeit: »Je l'avoue avec complaisance, mais avec une juste assurance, Louis XIII., ne fut point gouverné.« AaO. S. 1237.

ZfP 59. Jg. 4/2012 
comparer ce roi ni son règne avec les deux précédents; hâtons-nous de tirer le rideau sur un tout si peu susceptible de ce parallèle. " 135

Es ist das miteinander Verbindende, dem sich Saint-Simon zuwendet. Sein dem Willen Gottes geschuldeter bedingungsloser Gehorsam ließ Ludwig XIII. sein von Heimsuchungen angefülltes Lebensende entgegensehen "avec la fermeté d'un héros" nachdem von diesem »chrétien par excellence" in der Erwartung eines gestrengen Richterspruches auch sein weltlicher Auftrag vorbildlich erfüllt wurde. ${ }^{136}$

Unruhen und Kriege haben Vater und Sohn nicht davon abgehalten, der Wohlfahrt des Landes aufzuhelfen, de "laisser leur royaume florissant, opulent, heureux" - eine stattliche Bilanz, die durch die Hinterlassenschaft des Nachfolgers in ihr Gegenteil verkehrt wurde: "Etat difforme et déplorable si différent en tout de celui où Henri IV et Louis XIII l'avaient laissé. «137

Der Blick auf das dem dritten Bourbonen in seinen letzten Lebenstagen Zugestoßene und dessen nachlassende Bewältigung fördert Einsichten zutage, die allenfalls der Resignation Raum gewähren: "l'ordre de sa cour, la conduite de sa famille, l'économie du gouvernement ne semblèrent plus le toucher, lui qui jusqu'alors en avait été si jaloux «. ${ }^{138}$

Die Begegnung mit dem Tod rückt den König in ein anderes Licht und Saint-Simon versagt es sich nicht seiner Festigkeit, seinem Gleichmut, seinem Gottvertrauen nunmehr Lob zu spenden: "Que de grandeur, que de fermeté, que de noblesse, que de piété, mais que d'ignorance, que d'erreurs ... dont il ne parut pas s'apercevoir. «139

Das lädt den Autor abermals ein, der Eigenart der Gattung entsprechend Profile abzustecken und mit sich vertiefendem Verständnis die Unterschiede unparteiisch und wahrheitsgemäß zu erkunden. ${ }^{140}$

So sieht Saint-Simon seine bisherige Einschätzung durch das testamentarische Vermächtnis Ludwig XIII. abermals bestätigt: »une disposition siparfaite, où tout ne respirait que le bien, l'avantage, le repos intérieur, et la plus sage, la plus simple, la plus juste administration d'Etat ", ${ }^{141}$ der es nicht obliegt, reumütig um Vergebung zu bitten. Denn sein Bild leuchtet in hellen Farben, »Louis XIII était irréprochable« und das göttliche Gebot war eine lebenslang beachtete Richtschnur, »la lampe qui avait éclairé ses pas « ${ }^{142}$

Eine alle bisherigen Unterschiede einebnende Begegnung beider Monarchen mit dem Tod wird von Saint-Simon als trügerisch verworfen. Deshalb verknüpft er seinem Gerechtigkeitsbedürfnis zuliebe "la fermeté, la tranquillité, la patience, la liberté d'esprit, la grandeur d'âme à prévoir et ordonner des choses les plus tristes" mit der Vater und Sohn

135 Saint-Simon, aaO. (FN27) S. 1264.

136 Saint-Simon, aaO. (FN27) S. 1265.

137 Saint-Simon, aaO. (FN27) S. 1277.

138 Saint-Simon, aaO. (FN27) S. 1281.

139 Saint-Simon, aaO. (FN27) S. 1287.

140 Saint-Simon, aaO. (FN27) S. 1288.

141 Saint-Simon, aaO. (FN27) S. 1290.

142 Saint-Simon, aaO. (FN27) S. 1291. 
zuzuschreibenden, jeweils eigenen Wahrheit. Quelle immense disparité donc, so die ernüchternde Feststellung des Beobachters »entre la mort du père et celle du fils«. ${ }^{143}$

Hier ein nach standhaft durchlittener schwerer Erkrankung in Würde vollzogener Abschied: "une mort si sainte, si grande, si héroique - dort eine vom geistlichen und persönlichen Umfeld unverhohlen zur Schau gestellte Gleichgültigkeit gegenüber dem Sterbenden. "Ce fut dans cet abandon que Louis XIV mourut. "144 Aber auch über seinen Tod herrscht Trauer bei denen, die seine oft unkenntlich gewordene Tugendhaftigkeit bezeugen können, seine Güte, seine Gerechtigkeits- und Wahrheitsliebe, »enfin cette fermeté d'âme et cette grandeur de courage" als Wegbegleiter bis zum Lebensende. An dieser Stelle muss sich Saint-Simon eingestehen, dass sein Anliegen an Grenzen stößt, "que la comparaison de ces trois morts ... est une chose tout à fait impossible .145

So tritt an die Stelle des Unvergleichbaren, die das Ganze überblickende Abwägung. Sie gestattet keinen $Z$ weifel an der für Saint-Simon sich verfestigenden "juste préférence de Louis XIII: le tout balancé fait magnifiquement sortir Louis XIII de ce tableau "146 - "la pureté imcomparable de ses moeurs, sa capacité militaire, modestie, piété« liefern dafür eine hinreichende Begründung.

Dem Vorgänger hält Saint-Simon zugute, dass er, von großer Menschenkenntnis unterstützt, einer Kernforderung frühneuzeitlicher Regierungslehre zu entsprechen vermochte, die den Herrscher dazu aufruft, die für effektives Regierungshandeln notwendigen besten Mitarbeiter auszuwählen, ohne damit seiner Autorität Abbruch zu tun. Das war unbestreitbar nach den Saint-Simon vertrauten Auffassungen »la sagesse et le nerf de ce beau règne". ${ }^{147}$

Die Suche nach lückenloser Wahrheit verbindet sich mit dem Bedürfnis dem bislang Wahrgenommenen durch wiederkehrende Annäherungen untrügliche Gewissheit zu verleihen: "J'ai fait tout ce qui a été en moi pour concilier ce qui ne se peut taire dans un

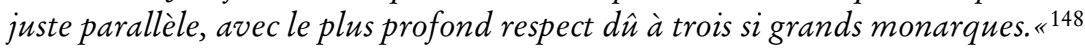

Bereits Vorgefundenes verdichtet sich nunmehr zu einer abschließenden Zusammenschau. In sie miteinbezogen wird die capacité militaire. Sie stand Heinrich IV. zu Gebote, aber nur der Sohne macht einen Vergleich zulässig durch seine Ebenbürtigkeit en valeur, en exploits, en science militaire, durch die Bereitschaft dem aus größerer Erfahrung herrührenden Ratschlag Gehör zu schenken. ${ }^{149}$

Nichvergleichbares galt es dagegen bereits zu vermelden hinsichtlich der freizügig gestalteten Liebesbeziehungen des Großvaters ${ }^{150}$ und derjenigen der einnehmenden Per-

143 Saint-Simon, aaO. (FN27) S. 1292.

144 Saint-Simon, aaO. (FN27) S. 1294.

145 Saint-Simon, aaO. (FN27) S. $1294 f$.

146 Saint-Simon, aaO. (FN27) S. 1298.

147 Saint-Simon, aaO. (FN27) S. 1302.

148 Saint-Simon, aaO. (FN27) S. 1308f.

149 Saint-Simon, aaO. (FN27) S. 1310.

150 Héroique jusque dans ses fautes, jusque dans ses vices, so das Urteil des homme politique De Lescure, zitiert bei Marylène Vincent, Henri IV et les femmes, Editions Sud Ouest, 2010, S. 243.

ZfP 59. Jg. 4/2012 
sönlichkeit des Enkels: "un grand et jeune roi ... n'échappe pas aisément à l'amour «. ${ }^{151}$ Einzigartig nimmt sich dagegen der hohe moralische Anspruch aus, dem sich Ludwig XIII. unterwarf, "quelle âme grande, forte sainte, que les mondains ne sont pas dignes d'admirer «. ${ }^{152}$ Jedoch verband die Absicherung des jeweiligen nach innen und außen gerichteten Machtanspruches Vater und Sohn miteinander. »La sagesse a manifestement présidé au gouvernement de Henri IV, plus encore à celui de Louis XIII. "153 Unter diesem Blickwinkel sieht sich der Autor nicht dazu aufgerufen, an der abschätzigen Bewertung der Regierungstätigkeit des Enkels Abstriche vorzunehmen.

Die Aufeinanderfolge von drei bedeutenden Herrschern verstand Saint-Simon als Einladung nach festgesetzten Kriterien, die ihr Handeln bestimmenden Charakterzüge zu erkunden. Das jeweils Eigene bietet dem Autor die Gelegenheit einer Wiedergutmachung, den Mittleren vom Makel eines nur als parfait automate ${ }^{154}$ Agierenden zu befreien. So ist am Ende der zurückgelegten Wegstrecke das abwägend Beobachtete zur Gewissheit herangereift. Unvergleichbar bleiben Erziehung und Herrschaftsantritt. Das von Ludwig XIII. willensmäßig Selbsterworbene steht in krassem Gegensatz zu dem von Ludwig XIV. Vorgefundenen. ${ }^{155}$

Zum Gegenstand der Bewunderung geraten die einzigartigen moeurs incomparables de Louis XIII. ${ }^{156}$ En vertu militaire wird er sich seinem Vater als ebenbürtig erweisen. „Ce fut donc à soi-même, que le jeune monarque dut tout, à sa valeur, à sa volonté, à son application, à s'instruire. "157 Die Einnahme von La Rochelle legt davon Zeugnis ab. »Ce sont deux héros bien reconnus pour tels, car le sublime $d u$ courage et la capacité militaire ne fut jamais disputée à Louis XIII. "158 Schließlich gerät die Todeserfahrung zum untrüglichen Prüfstein für den dem srobur animi verpflichteten Wertekanon des Autors. War das Verwobensein von unbezähmbarer Leidenschaft ${ }^{159}$ mit dem abgründigen Todesschicksal des Vaters von Trauer und Entsetzen begleitet, so gewährt das Sterben des Sohnes einer letzten Bewährungsprobe Raum: »quelle fermeté d'âme, quelle grandeur de courage, quelle aimable paix, quels élans d'espérance et de désir d'aller à Dieu ". ${ }^{160}$ Und endlich wird es der an den altersmüden Enkel herantretende Tod sein, der das Trennende tilgt: "La seule chose qui puisse être comparée dans la fin des ces deux monarques est la fermeté, la tranquillité, la patience, l'inaltérable paix avec lesquelles ils virent, ils goûtèrent, ils attendirent la mort. «161

151 Saint-Simon, aaO. (FN27) S. 1312.

152 Saint-Simon, aaO. (FN27) S. 1314.

153 Saint-Simon, aaO. (FN27) S. 1317.

154 Saint-Simon, aaO. (FN27) S. 1323.

155 Saint-Simon, aaO. (FN27) S. 1324.

156 Saint-Simon, aaO. (FN27) S. 1325.

157 Saint-Simon, aaO. (FN27) S. 1327.

158 Saint-Simon, aaO. (FN27) S. 1327.

159 »Henri IV, lorsque la passion le tenait, devenait l'inconscience même, atteste un contemporain.«Zitiert bei M. Vincent, Henri IV et les femmes, aaO. (FN) S. 243.

160 Saint-Simon, aaO. (FN27) S. 1331.

161 Saint-Simon, aaO. (FN27) S. 1323. 


\section{Schluss}

Zwei herausragende, in unterschiedlichen Lebenswelten beheimatete Vertreter ihres Zeitalters, der deutsche Universalgelehrte G. W. Leibniz und der Ducmémorialiste SaintSimon (1675-1755) ${ }^{162}$ bezeugen aus unterschiedlichem Anlass das Fortleben einer auf praktische Nutzbarmachung hin angelegte, als persönlichen Appell gestaltete römischstoische Tugend- und Pflichtenlehre in einer, wie Oestreich bemerkt, sehr auf die Person des Fürsten, des Staatsmannes und Beamten, des Feldherrn und Soldaten zugeschnittenen Form. ${ }^{163}$

Um der die Freiheit der Nation Allemande bedrohenden französischen Übermacht zu begegnen, hält es Leibniz für geboten, das Erfolgsgeheimnis des Gegners zu ergründen. Es kommt zum Vorschein in den von der prudence militaire inspirierten Edikten aus der fabrique du Cardinal Richelieu, die zum wachrüttelnden Vorbild gereichen sollten. Um dem militärischen Machtgefälle Einhalt zu gebieten ist also, so mahnt der Autor, von vertu militaire beflügelte, mutige Entschlossenheit nötig, gepaart mit der ernsthaft betriebenen Umsetzung der die Kampftauglichkeit und sittliche Ertüchtigung befördernden militärischen Klugheitsregeln.

»Von unbedingter Wahrheitsliebe und Dankbarkeit angetrieben, ergreife ich die Feder«, erklärt eingangs Saint-Simon um ein durch ingratitude und ignorance verzerrtes Bild zu korrigieren, um seinem Favoriten dem den Ehrentitel le Juste tragenden Ludwig XIII. Gerechtigkeit widerfahren zu lassen, ihn zu rehabilitieren. Die Entdeckung des Verborgenen schafft Raum für die Wahrnehmung des Außergewöhnlichen: "L'adorable monarque couronna l'héroisme, illustrant splendidement et indestructiblement la catégorie de l'Idéal.«164 Die Erfüllung dieses Wunsches vollzieht sich nicht zuletzt vor dem Hintergrund einer beachtlichen culture livresque ${ }^{165}$ wobei die über den Anteil an livres

162 M. Fumaroli, Les m'moires du XVIIe siècle au carrefour des genres en prose, in: XVIIe siècle, Paris 1971, S. 37 bilanziert einen Übergang von der militärisch-politisch geprägten Gattung zu derjenigen des gens de cour où se forme et se polit le meilleur de l'esprit français.

163 G. Oestreich, aaO. (FN4) S. 78. I. Flandrois, L'institution du Prince, 1992, S. 184, hebt die dafür ausschlaggebende Bedeutung von prudence und vertu hervor, allerdings ohne den begrifflichen Hintergrund näher zu beleuchten. Toute institution du Prince - une fonction nécessaire de transmission des valeurs morales. AaO. S. 218. Beispielgebend für anonym bezeugte neustoische Einflüsse ist der "Traité de morale pour l'Education des Princes, tiré des Peintures de la Gallerie de Saint Cloud de l'abbé Laurent Morelat, aumonier de Monsieur le Duc d'Orléans. Mit den allegorisch dargestellten vier Jahreszeiten verbinden sich ihnen eigentümlich erscheinende Tugenden, so etwa constance, fermeté invincible face aux adversités inséparables de la grandeur als dem Winter zugehörig. Olivier Leplatre, Ekphrasis et politique: Le traité de morale ... de l'abbé L. Morelet, in: Discours politique et genres littéraires, XVIe - XVIIe siècles. Textes réunis par S. Gruffat et O. Leplatre. Cahiers Du Gadges, No. 6, Genève 2008, S. 290ff.

164 Y. Coirault, aaO. (FN27) S. XIX. Einen Zusammenhang von »véritable culte«und »curiosité«bei Saint-Simon vermerkt F. Briot, Usage du monde, usage de soi. Enquête sur les mémorialistes d'ancien Régime, 1994, S. 156 u. $245 \mathrm{ff}$.

165 D. van der Cruysse, La bibliothèque du Duc de Saint-Simon. In: XVIIe siècle, Paris 1971, Bd 94/95, verweist auf den nur unvollständig überlieferten Buchbestand.

ZfP 59. Jg. 4/2012 
outils véritables ${ }^{166}$ vermittelte sittlich moralische Verankerung des frühneuzeitlichen Politikverständnisses eines "nostalgique d'un ancien monde" (Coirault) sowie ihre Geltendmachung anlässlich der parallelen Betrachtung dreier aufeinanderfolgender Herrscherpersönlichkeiten nicht von der Hand zu weisen ist, zumal auch profunder Sachverstand der Frage nicht auszuweichen vermag: »Cette constance, au moins extérieure, cette identité à soi-même malgré tout, n'est-ce pas, au fond, la vertu maîtresse et le premier principe de l'éthique ... et de la théologie politique de notre duc et pair? «167

\section{Zusammenfassung}

Fas est ab hoste doceri - es dem überlegenen Gegner gleichzutun, dazu fordert 1694 der Universalgelehrte Leibniz seine »Nation Allemande" auf, indem er die Aufmerksamkeit auf die "fabrique de Richelieu " lenkt, die Hilfestellung leistet beim Aufbau eines stehenden Heeres, das, errichtet auf der Grundlage einer erneuerten neustoisch geprägten »disciplina militaris«, zum Träger des neuzeitlichen Machtstaates aufrückte. Das Fortleben des stoisierenden Moralismus bekundet nicht minder überzeugend der einer "rébabilitation" der Person Ludwigs XIII. geschuldeten »Parallèle des trois premiers Rois Bourbons" des Duc de Saint-Simon. Der Ludwig XIII. zugesprochene, von "sainteté" überhöhte »héroisme " gründet in einer auch vom Kardinalpremier nachhaltig inspirierten, der römischen Stoa verpflichteten Morallehre als ein »le fond de sa pensée ou de son paysage intérieur" (Coirault) nachhaltig befruchtendes Ferment.

\section{Summary}

In 1694, universal scholar Leibniz called upon his "Nation Allemande" to learn from its superior opponent - fas est ab hoste doceri - by drawing attention to the »fabrique de Richelieu «, which offers assistance with establishing a standing army which, created on the basis of a renewed »disciplina militaris" influenced by Neostoicism, became the foundation for the modern authoritarian state. The continued existence of stoical moralism is expressed no less convincingly by the duc de Saint-Simon in his »Parallele des trois premiers Rois Bourbons", aimed at a "rébabilitation" of Louis XIII. The "héroisme" attributed to Louis XIII, exaggerated as it is by "sainteté", has its roots in a moral doctrine which was also lastingly inspired by the cardinal premier and is dedicated to Roman Stoicism, as a ferment which enduringly fertilizes »le fond de sa pensée ou de son paysage intérieur« (Coirault).

Carl Siedschlag, Investigating the Development of Authoritarian and Military Views in Leibniz and Saint-Simon

166 Y. Coirault, Un»Tacite inculte«? Saint-Simon et les »belles lettres « in: De l'Estoile à SaintSimon, recherche sur la culture des mémorialistes au temps des premiers rois Bourbons. Actes et Colloques, 43, 1993, S. 134 u. Anm. 11.

167 Y. Coirault in: Saint-Simon, Mémoires V, Pléiade, S. 1440, Anm. 2. 